\title{
Bacterial Pneumonias during an Influenza Pandemic: How Will We Allocate Antibiotics?
}

Sandro K. Cinti, Andrew R. Barnosky, Steven E. Gay, Susan Dorr Goold, Marie M. Lozon, Kristin Kim, Phillip E. Rodgers, Nancy M. Baum, Bruce A. Cadwallender, Curtis D. Collins, Carrie M. Wright, and Robert A. Winfield

We are currently in the midst of the $2009 \mathrm{H} 1 \mathrm{~N} 1$ pandemic, and a second wave of flu in the fall and winter could lead to more hospitalizations for pneumonia. Recent pathologic and historic data from the 1918 influenza pandemic confirms that many, if not most, of the deaths in that pandemic were a result of secondary bacterial pneumonias. This means that a second wave of $2009 \mathrm{H} 1 \mathrm{~N} 1$ pandemic influenza could result in a widespread shortage of antibiotics, making these medications a scarce resource. Recently, our University of Michigan Health System (UMHS) Scarce Resource Allocation Committee (SRAC) added antibiotics to a list of resources (including ventilators, antivirals, vaccines) that might become scarce during an influenza pandemic. In this article, we summarize the data on bacterial pneumonias during the 1918 influenza pandemic, discuss the possible impact of a pandemic on the University of Michigan Health System, and summarize our committee's guiding principles for allocating antibiotics during a pandemic.

$\mathrm{T}$ HE CURRENT INFLUENZA PANDEMIC began with the simultaneous emergence of a new strain of $\mathrm{H} 1 \mathrm{~N} 1$ influenza in Mexico and in the U.S. in April 2009. ${ }^{1,2}$ While the first wave of pandemic $2009 \mathrm{H} 1 \mathrm{~N} 1$ influenza has been mild, a second wave this coming fall and winter could be more severe. This outbreak and the ongoing outbreak of $\mathrm{H} 5 \mathrm{~N} 1$ influenza in Southeast Asia have reawakened fears of a worldwide influenza pandemic of the sort that occurred in 1918. ${ }^{3}$ The so-called Spanish flu raged throughout the world, killing 40 to 50 million people in less than a year; an estimated 500,000 deaths occurred in the U.S. ${ }^{3}$

In recent years, the U.S. Department of Health and Human Services (HHS) and the individual states have launched substantial efforts to prepare for such an event. ${ }^{4}$

Sandro K. Cinti, MD, is Associate Professor, Infectious Diseases, University of Michigan Hospitals/VA Ann Arbor Health Systems. Andrew R. Barnosky, DO, MPH, is Associate Professor, Department of Emergency Medicine, Program in Bioethics, University of Michigan Medical School and Medical Center. Steven E. Gay, MD, MS, is Assistant Professor, Department of Internal Medicine, and Director of Critical Care Support Services, University of Michigan Health System. Susan Dorr Goold, MD, MHSA, MA, is Professor of Internal Medicine and Director, Bioethics Program, University of Michigan Medical School. Marie M. Lozon, MD, is Division Director, Pediatric Emergency Medicine, Departments of Emergency Medicine and Pediatrics, University of Michigan Health System. Kristin Kim, MD, PhD, is a Pediatric Emergency Medicine Fellow, Department of Emergency Medicine, University of Michigan Health System. Phillip E. Rodgers, MD, is Assistant Professor, Department of Family Medicine, University of Michigan. Nancy M. Baum, MHS, is a doctoral candidate, School of Public Health, University of Michigan. Bruce A. Cadwallender, MS, is Director of Safety and Emergency Management, Safety Management Services, University of Michigan Hospitals and Health Centers. Curtis D. Collins, PharmD, MS, is Clinical Pharmacist, Infectious Diseases Department of Pharmacy Services, University of Michigan Health System. Carrie M. Wright, MS, is an Emergency Management Specialist, University of Michigan Hospitals and Health Centers. Robert A. Winfield, MD, is Chief Health Officer and Director of the University Health Service, Office of the President and Division of Student Affairs, University of Michigan. All are in Ann Arbor, Michigan. 
One element of preparedness that has been particularly challenging is determining how scarce resources, such as influenza vaccine, antiviral medication, and ventilators, will be allocated. Although many of these resources are being stockpiled by the states, the federal government, and private entities, it is unlikely that stockpiling will be sufficient to avoid severe scarcity in a pandemic. As a result, HHS and other entities have developed guidelines and priority lists for antivirals, ${ }^{4}$ vaccine, ${ }^{4}$ and ventilators. ${ }^{5,6}$ However, little attention has been paid to antibiotics as a scarce resource during a pandemic; there are 2 reasons for this.

First, as a result of bioterrorism preparedness efforts, Congress established a Strategic National Stockpile (SNS) containing antibiotics for Category A biological agents, ${ }^{7}$ and this stockpile may have created a false sense of security about the availability of antibiotics during a pandemic. Unfortunately, the SNS antibiotics are not as effective against postinfluenza bacterial pneumonias. ${ }^{8}$ For example, ciprofloxacin, a major component of the SNS, has poor activity against community-acquired pneumonia (CAP) bacteria including Staphylococcus aureus and Streptococcus pneumoniae, 2 common postinfluenza pathogens. ${ }^{7}$ Doxycycline, another SNS antibiotic, is recommended only for outpatient therapy of community-acquired pneumonia. $^{8}$

Second, most of the mortality and morbidity attributed to the 1918 influenza virus was initially believed to be mediated through a "cytokine storm," the body's immune response to overwhelming viral infection. ${ }^{9} \mathrm{H} 5 \mathrm{~N} 1$ infection is thought to have a similar disease mechanism. ${ }^{10}$ This type of response is best handled either by preventing infection with vaccine or by giving antivirals when disease occurs. However, a recent review of historic and pathologic data from the 1918 pandemic has put the "cytokine storm" theory in doubt and, instead, suggests that most deaths were a result of secondary bacterial infections. ${ }^{11}$ Thus, the role of antibiotics in mitigating the effects of an influenza pandemic becomes paramount.

In December 2007, the University of Michigan Health System's (UMHS) Pandemic Planning Committee established the Scarce Resource Allocation Committee (SRAC) to develop guiding principles for allocating scarce resources in an equitable fashion during an influenza pandemic. The SRAC is composed of experts in ethics, infectious diseases, pediatrics, internal medicine, intensive care medicine, emergency medicine, pharmacy, palliative care, public health, and emergency management; the committee has been meeting monthly since its inception. The SRAC recommendations encompass several other potential scarce resources, including ventilators, oxygen, antivirals, vaccine, and staff; however, this article will focus on our recommendations for antibiotic allocation. This article is not meant to be an exhaustive review of the diagnosis and treatment of bacterial pneumonias, as these topics are covered elsewhere in the literature. We have included sections on palliative care and public engagement because these are germane to any discussion about scarce resources.

\section{Bacterial Pneumonias and Influenza}

Influenza can predispose a patient to bacterial pneumonias, and it has been estimated that approximately $25 \%$ of deaths during the influenza season are due to this complication. ${ }^{12}$ Current evidence suggests that infection with influenza virus alters the tracheobronchial epithelium and damages cilia, thereby providing an environment favorable to bacterial pathogens. ${ }^{13}$ The most common pathogens associated with postinfluenza bacterial pneumonia include Streptococcus pneumoniae, Staphylococcus aureus, and Haemophilus influenza. ${ }^{14}$ Community-acquired methicillin-resistant Staphylococcus aureus (CA-MRSA) has recently emerged as a significant problem, and an increase in Staphylococcus aureus-associated deaths following influenza infection has been noted in children. ${ }^{15}$ The Infectious Diseases Society of America (IDSA) and the American Thoracic Society (ATS) have developed guidelines for the treatment of community-acquired pneumonia, including postinfluenza pneumonias. ${ }^{8}$ Although the specifics of these guidelines are beyond the scope of this article, our committee used them to develop recommendations for allocating antibiotics during a pandemic.

Morens et al. recently reported the results of their study looking at preserved lung tissue from 58 victims of the 1918 influenza pandemic. ${ }^{11}$ They also reviewed pathologic and bacteriologic data from 109 studies that described more than 8,000 autopsies of 1918 influenza victims. ${ }^{11}$ The pathologic characteristics of the 58 lung specimens were consistent with bacterial pneumonia, demonstrating neutrophilic infiltrates in the alveoli. The bacteriologic data from 96 series showed that more than $95 \%$ of lung cultures had bacteria in them. The authors concluded that the majority of influenza deaths in the 1918 pandemic were caused by secondary bacterial infections. ${ }^{11}$ Brundage and Shanks reviewed data on the epidemiologic and clinical characteristics of the influenza victims during the 1918 pandemic. ${ }^{16}$ They found that most deaths occurred over a period of 7 to 10 days and sometimes more than 2 weeks after the onset of symptoms. Also, most pneumonia cases had bacterial pathogens recovered, and experts of the day concurred that secondary pneumonias were causing death. The authors concluded that bacterial pneumonias were responsible for most deaths. ${ }^{16}$

\section{Potential Impact of an Influenza Pandemic on UMHS}

The University of Michigan Health System Influenza Pandemic Preparedness Group used assumptions developed by HHS to estimate the impact an influenza pandemic would have on UMHS. ${ }^{17}$ For a population of 450,000 people (UMHS catchment) with an infection rate of $25 \%$, UMHS would have the following level of patient activity over 8 to 12 weeks:

Biosecurity and Bioterrorism: Biodefense Strategy, Practice, and Science 
- 57,000 outpatient visits

- 12,500 hospitalizations

- 1,900 ICU admissions

- 1,700 deaths (3\% mortality rate)

It has been estimated that $15 \%$ to $20 \%$ of influenza patients developed pneumonia during the 3 pandemics of the $20^{\text {th }}$ century. ${ }^{18,19}$ Thus, of the 57,000 outpatients who present ill with influenza, at least 11,400 can be expected to develop bacterial pneumonia at some point during their illness. If each patient requires at least 7 days of antibiotic therapy (some will require more), UMHS will need approximately 80,000 doses of antibiotics appropriate to treating pneumonia. Of the 11,400 patients requiring antibiotics for pneumonia, approximately 4,500 will be inpatients and will likely need several doses of intravenous antibiotics. The cost of stockpiling enough antibiotics to cover all these patients for 7 days is estimated to be over $\$ 600,000$ and is prohibitive. Stock rotations to manage expiration dates would be an additional burden. Furthermore, UMHS cannot rely on the SNS to provide enough antibiotics, because the program does not stock enough antibiotics or the appropriate antibiotics to cover postinfluenza pneumonias. ${ }^{7}$ Given these circumstances, the UMHS Scarce Resource Allocation Committee developed guiding principles for allocating antibiotics.

\section{Guiding Principles for Allocating Antibiotics during a Pandemic}

\section{Ethical Framework}

In developing guiding principles for allocating scarce resources during a pandemic, the Scarce Resource Allocation Committee adhered to the following ethical framework, which draws on the literature in ethics and public health: ${ }^{20-22}$

- Professional obligations to individual patients;

- Professional and institutional obligations of competence;

- Professional and institutional obligations of honesty and transparency;

- Distributive justice, including equal treatment, utility;

- Fair procedures, including in planning and implementation; and

- Accountability and legitimacy.

Ethical principles in medicine dictate that a clinician and a healthcare institution must provide competent care for individual patients. By this principle, it would be unethical for a clinician or hospital to withhold antibiotics from a patient diagnosed with bacterial pneumonia. However, the principle of distributive justice dictates that resources be allocated equitably in a population; therefore, changing antibiotic usage guidelines or even denying antibiotics in certain situations would be required during a shortage. In order to balance the individual good with that of a larger population, fair procedures must be in place well before a shortage of antibiotics occurs, and these procedures must be transparent to the public. An alternative allocation system whereby antibiotics are distributed on a first-come-firstserve basis until they are gone was felt by committee members to be unacceptable.

Based on obligations of competence, equal treatment, and utility, the committee developed strict prohibitions against the prophylactic use of antibiotics to prevent bacterial pneumonia. Only suspected or proven pneumonia warrants the use of scarce antibiotics. Certain patients at high risk for mortality from bacterial pneumonia (eg, those with COPD, renal failure, congestive heart failure, or who are immunocompromised) might be considered exceptions if antivirals fail to prevent worsening of respiratory symptoms. Generally, antibiotics should be allocated to the sickest patients and to those who have the greatest likelihood for survival. The committee, therefore, addressed antibiotic use in the intensive care unit (ICU), the hospital (including off-site wards), and the ambulatory care setting.

\section{Inpatients and ICU Patients}

For the sickest adult inpatients (ICU/ventilated patients), we recommend distributing antibiotics based on Sequential Organ Failure Assessment (SOFA) scores. A SOFA score is a rapid method of assessing survival; it relies on oxygenation, blood pressure, platelet count, bilirubin, creatinine, and Glasgow Coma Score. ${ }^{23}$ An initial SOFA score is calculated at admission and again at 48 and 96 hours. An initial SOFA score of $>11$ predicts a mortality rate of $95 \%$; a SOFA score $<9$ predicts a mortality rate of less than $33 \% .^{23}$ At 48 hours, the best predictor of mortality was an increase $(>50 \%$ mortality) or decrease $(<27 \%$ mortality) in the SOFA score.

The SOFA score has been proposed by several groups as a way to allocate ventilators during an influenza pandemic. ${ }^{4,24}$ We propose that patients with a SOFA score of $>11$ should not receive antibiotics if these are in short supply; these patients would be considered to require palliative care. In the ventilator allocation protocol, patients with a SOFA score $>11$ are not eligible to be placed on a ventilator given that they would have a $>95 \%$ mortality rate. Our committee felt that such a high mortality should also preclude administration of antibiotics if they are a scarce resource.

ICU patients with SOFA scores $\leq 11$ and other hospitalized patients should be eligible for antibiotic treatment but only when pneumonia is highly suspected or proven based on clinical symptoms, radiologic procedures, and laboratory data. Patients who are removed from ventilators and/or placed on a palliative care protocol will no longer receive antibiotics if they are a scarce resource.

Clinical case definitions of bacterial pneumonia are available in the literature and will be crucial in allocating 
antibiotics. ${ }^{8,25}$ Early in a pandemic, antibiotics and diagnostic tests will be plentiful, but this is the time when strict adherence to bacterial pneumonia diagnostic and treatment protocols will be essential. Appropriate antibiotic usage will delay a scarcity of this resource. During the first wave of the 2009 H1N1 influenza pandemic, the UMHS Pandemic Committee developed order sets for inpatients and ICU patients admitted for flu. These order sets included protocols for the diagnosis and treatment of bacterial pneumonias. While bacterial pneumonias have not been a significant problem in the first wave, these protocols will be in place should the situation worsen in the fall.

It is expected that, as the pandemic progresses, diagnostic and treatment protocols will have to be modified. Clinicians will have fewer diagnostic tools (eg, chest X-ray, blood tests) and may have to rely solely on symptoms and exam findings when making decisions on the need for antibiotics. Furthermore, the scarcity of certain antibiotics will necessitate the use of other antibiotics previously considered second and third line (see Choice of Antibiotics below).

\section{Outpatients}

The outpatient setting presents a particularly daunting challenge for allocating antibiotics, because the huge number of patients and the need for rapid triage will tend to favor overuse of antibiotics. The committee was concerned that large numbers of people might inappropriately get antibiotics, including the "worried well," or people who have no infection, and those with viral symptoms from influenza or other viruses who do not have a bacterial infection. In the past, education efforts to reduce inappropriate antibiotic use in the ambulatory care setting have been effective, ${ }^{26}$ but these will have to be instituted early and aggressively during a pandemic. Clinical case definitions and guidelines for outpatient therapy should be developed before a pandemic occurs, and these protocols should be vetted by multiple clinicians.

\section{Pediatric Patients}

The committee felt that more attention must be paid to the pediatric population in regards to antibiotic allocation. SOFA scores may not be an accurate measure of survival in this population. Some experts have proposed using the Pediatric Logistic Organ Dysfunction (PELOD) scoring system for allocation of ventilators. ${ }^{27}$ This scoring system relies on assessment of 7 organ systems and requires minimal lab data (ie, creatinine, arterial blood gas [ABG], white blood count [WBC], platelet count [plt] and aspartate aminotransferase [AST], prothrombin time [PT]). A PELOD score of $>26$ predicts $100 \%$ mortality in a pediatric patient, and such a score would make the patient ineligible for antibiotics in accordance with our guiding principles for adults with poor SOFA scores. Also, different antibiotics and different doses are required in the pediatric population. Several common antibiotics for pneumonia, including flouroquinolones and tetracyclines (doxycycline) are problematic in children because of their effects on bones and tendons. ${ }^{28,29}$ These can be used if no other antibiotics are available, but special protocols will need to be in place to guide and protect physicians who administer them.

\section{Choice of Antibiotics}

Antibiotic choice based on price and availability will be inevitable during an influenza pandemic. The use of cheaper and more available oral antibiotics like doxycycline, ciprofloxacin, and amoxicillin will be necessary in the outpatient setting, even if these are less effective than intravenous antibiotics (eg, ceftriaxone, vancomycin) and more expensive oral antibiotics (eg, moxifloxacin, linezolid). Ciprofloxacin and doxycycline may be more available because they are stocked in the SNS and other repositories such as the Veteran's Affairs Medical Center Caches. ${ }^{7,30}$ Also, in the inpatient setting, a more rapid transition from intravenous (IV) to oral antibiotics may need to occur both because of low availability of IV antibiotics and a need to discharge patients quickly to free up hospital bed space. The committee felt strongly that antibiotic protocols should be developed well ahead of a pandemic in order to guide clinicians both in the hospital and in the outpatient setting.

The committee agreed that beyond prioritizing antibiotics for patients who have a proven or suspected pneumonia and are likely to survive, it does not make sense to stratify people further. Patients with bacterial pneumonias who go untreated are very likely to get worse and die. Denying antibiotics to anyone in this situation seems ethically unsound if that person is likely to survive with the treatment. This is in contrast to the use of antivirals. Antivirals, as treatment, would be used in patients with influenza symptoms regardless of the presence of pneumonia. Prioritizing these drugs can be justified because most people $(97 \%)$ are expected to survive influenza in a 1918-like scenario with no treatment. ${ }^{17,31}$ Antiviral treatment is most likely to be helpful in high-risk groups; thus, HHS has developed a priority list for these drugs. ${ }^{4}$

\section{Palliative Care}

Although the Scarce Resource Allocation Committee's antibiotic allocation strategy does not support the use of antibiotics for patients who are deemed to be in need of palliative care, our committee affirmed an ethical duty to provide aggressive symptom control and support for all patients, regardless of survival likelihood. Palliative care protocols will be the key intervention for those expected to die and should include treatment of dyspnea and related 
anxiety, pain, and other physical symptoms caused by the underlying illness, as well as psychological, spiritual, and family counseling to the degree possible. Expert interdisciplinary palliative care teams are now present in a majority of U.S. hospitals to provide such care routinely, but they are themselves a scarce resource given that so few of these experts are present at any particular hospital.

Our committee has proposed implementation of systemwide pandemic palliative care guideline, based on accepted national quality standards. ${ }^{32}$ Key guideline priorities include developing protocols for symptom relief to be used by first-line providers, developing strategies for deploying palliative care personnel, and ensuring adequate supplies of essential medications, particularly opioids and benzodiazepines, to relieve dyspnea, pain, anxiety, and terminal restlessness. The fundamental goal is to ensure that patients get the highest-quality, most appropriate care possible throughout their course of illness.

\section{Public Engagement about Scarce Resource Allocation}

Public education, discussions, and deliberations about scarce resource allocation are critical and must occur before the current pandemic becomes more widespread. Healthcare facilities and public health and emergency management organizations in a county, region, and state must agree on when and how standards of care will be altered during a pandemic and how resources can best be allocated. Regarding antibiotic use, changes in established protocols and guidelines will have to be accepted and followed by all caretakers in a community. Any appearance of providing different levels of care could undermine public health efforts to slow the pandemic.

The public must also have a role in discussions about scarce resource allocation. There is an expectation in our country that antibiotics are readily available. In fact, many patients demand these medications for any upper respiratory infection. ${ }^{33}$ Educating the public about the possibility of antibiotic shortages during a pandemic and about how public health will respond to these shortages is essential to prepandemic planning.

The UMHS Scarce Resource Allocation Committee intends to have ongoing discussions with regional and state entities about allocation issues. As we develop our scarce resource allocation plan, drafts of our document will be shared for comment and revision with our state Pandemic Ethics Committee and the county Health Emergency Response Committee (HERC), which includes representatives from public health, all area hospitals, emergency management, the school system, social work agencies, law enforcement agencies, and the Red Cross. Furthermore, we are working with our county public health partners to develop a forum for public discussion on scarce resource allocation. Such a gathering has already been convened at the national level by HHS and has proven to be very useful for planning. ${ }^{34}$

\section{REFERENCES}

1. U.S. Centers for Disease Control and Prevention (CDC). Swine-origin influenza A (H1N1) virus infections in a school-New York City, April 2009. MMWR Morb Mortal Wkly Rep 2009 May 8;58(17):470-472.

2. U.S. Centers for Disease Control and Prevention (CDC). Outbreak of swine-origin influenza A (H1N1) virus infection-Mexico, March-April 2009. MMWR Morb Mortal Wkly Rep 2009 May 8;58(17):467-470.

3. Taubenberger JK, Reid AH, Fanning TG. Capturing a killer flu virus. Sci Am January 2005:62-71.

4. U.S. Department of Health and Human Services. HHS Pandemic Influenza Plan. http://www.hhs.gov/pandemicflu/ plan/. Accessed August 13, 2009.

5. Hick JL, O'Laughlin DT. Concept of operations for triage of mechanical ventilation in an epidemic. Acad Emerg Med 2006;13:223-229.

6. Powell T, Christ KC, Birkhead GS. Allocation of ventilators in a public health disaster. Disaster Med Public Health Prep 2008;2:20-26.

7. Esbitt D. The Strategic National Stockpile: roles and responsibilities of health care professionals for receiving the stockpile assets. Disaster Manag Response 2003;1:68-70.

8. Mandell LA, Wunderink RG, Anzueto A, et al. Infectious Diseases Society of America/American Thoracic Society consensus guidelines on the management of communityacquired pneumonia in adults. Clin Infect Dis 2007;44(Suppl 2):S27-S72.

9. Tumpey TM, García-Sastre A, Taubenberger JK, et al. Pathogenicity of influenza viruses with genes from the 1918 pandemic virus: functional roles of alveolar macrophages and neutrophils in limiting virus replication and mortality in mice. L Virol 2005;79:14933-14944.

10. Wong SS, Yuen KY. Avian influenza virus infections in humans. Chest 2006;129:156-168.

11. Morens DM, Taubenberger JK, Fauci AS. Predominant role of bacterial pneumonia as a cause of death in pandemic influenza: implications for pandemic influenza preparedness. Infect Dis 2008;198:962-970.

12. Simonsen L. The global impact of influenza on morbidity and mortality. Vaccine 1999;17(Suppl 1):S3-S10.

13. Levandowski RA, Gerrity TR, Garrard CS. Modifications of lung clearance mechanisms by acute influenza A infection. ILab Clin Med 1985;106:428-432.

14. Schwarzmann SW, Adler JL, Sullivan RJ Jr, et al. Bacterial pneumonia during the Hong Kong influenza epidemic of 1968-1969. Arch Intern Med 1971;127:1037-1041.

15. Finelli L, Fiore A, Dhara R, et al. Influenza-associated pediatric mortality in the United States: increase of Staphylococcus aureus coinfection. Pediatrics 2008;122:805-811.

16. Brundage JF, Shanks GD. Deaths from bacterial pneumonia during 1918-19 influenza pandemic. Emerg Infect Dis 2008;14(8):1193-1199.

17. U.S. Department of Health and Human Services. HHS Pandemic Influenza Plan. Part 1: Strategic Plan. http://www. 
hhs.gov/pandemicflu/plan/part1.html. Accessed August 13, 2009.

18. Brundage JF. Interactions between influenza and bacterial respiratory pathogens: implications for pandemic preparedness. Lancet Infect Dis 2006;6:303-312.

19. Soper GA. The pandemic in the Army camps. JAMA 1918;71:1899-1909.

20. Levin PJ, Gebbie EN, Qureshi K. Can the health-care system meet the challenge of pandemic flu? Planning, ethical, and workforce considerations. Public Health Rep 2007;122:573578.

21. Rubinson L, Nuzzo JB, Talmor DS, et al. Augmentation of hospital critical care capacity after bioterrorist attacks or epidemics: recommendations of the Working Group on Emergency Mass Critical Care. Crit Care Med 2005;33: 2393-2403.

22. Kass NE, Otto J, O'Brien D, et al. Ethics and severe pandemic influenza: maintaining essential functions through a fair and considered response. Biosecur Bioterror 2008;6:227-236.

23. Ferreira FL, Bota DP, Bross A, et al. Serial evaluation of the SOFA score to predict outcome in critically ill patients. IAMA 2001;286:1754-1758.

24. Christian MD, Hawryluck L, Wax RS, et al. Development of a triage protocol for critical care during an influenza pandemic. CMAJ 2006;175:1377-1381.

25. Plouffe JF, Martin DR. Pneumonia in the emergency department. Emerg Med Clin North Am 2008;26:389-411.

26. Ranji SR, Steinman MA, Shojania KG, et al. Interventions to reduce unnecessary antibiotic prescribing: a systematic review and quantitative analysis. Med Care 2008;46:847862.

27. Leteurtre S, Martinot A, Duhamel A, et al. Development of a pediatric multiple organ dysfunction score: use of two strategies. Med Decis Making 1999;19:399-410.
28. Velissariou IM. The use of fluoroquinolones in children: recent advances. Expert Rev Anti Infect Ther 2006;4:853-860.

29. Sloan B, Scheinfeld N. The use and safety of doxycycline hyclate and other second-generation tetracyclines. Expert Opin Drug Saf 2008;7:571-577.

30. Teeter DS, Koenig KL. VA's role in bioterrorism preparations. Am J Infect Control 2000;28:321.

31. Nguyen-Van-Tam JS, Hampson AW. The epidemiology and clinical impact of pandemic influenza. Vaccine 2003;21: 1762-1768.

32. National Consensus Project for Quality Palliative Care. http://www.nationalconsensusproject.org/. Accessed August 13, 2009.

33. McNulty CA, Boyle P, Nichols T, et al. The public's attitudes to and compliance with antibiotics. Intimicrob Chemother 2007;60(Suppl 1):i63-i68.

34. Citizen Voices on Pandemic Flu Choices: A Report of the Public Engagement Pilot Project on Pandemic Influenza. http:// www.pandemicflu.gov/plan/federal/pepppimaintext.pdf. Accessed August 13, 2009.

Manuscript received May 12, 2009;

accepted for publication August 10, 2009.

Address correspondence to:
Sandro Cinti, MD
Associate Professor
Infectious Diseases
University of Michigan Hospitals/VA Ann Arbor Health Systems
2215 Fuller Rd.
Ann Arbor, MI 48105
E-mail: scinti@umich.edu

E-mail: scinti@umich.edu 
This article has been cited by:

1. Michael Eisenhut . 2009. Procalcitonin Measurement to Reduce Antibiotic Use in InfluenzaProcalcitonin Measurement to Reduce Antibiotic Use in Influenza. Biosecurity and Bioterrorism: Biodefense Strategy, Practice, and Science 7:4, 453-454. [Abstract] [Full Text] [PDF] [PDF Plus] 